\title{
Hacia un científico digital
}

\author{
Quercus Hernández ${ }^{1}$, Alberto Badías ${ }^{1}$, David González ${ }^{1}$, Francisco Chinesta ${ }^{2}$, Elías \\ Cueto $^{1}$ \\ ${ }^{1}$ Applied Mechanics and Bioengineering (AMB) \\ Instituto de Investigación en Ingeniería de Aragón (I3A) \\ Universidad de Zaragoza, Mariano Esquillor s/n, 50018, Zaragoza, Spain. \\ Tel. +34-976762707, e-mail: quercus@unizar.es \\ ${ }^{2}$ ENSAM (ParisTech)
}

\section{Resumen}

En este trabajo se pretende aplicar inteligencia artificial para predecir la evolución temporal de un sistema físico arbitrario sólo mediante el uso de datos, es decir, sin conocer las ecuaciones que lo rigen. Este método se ha validadado con dos sistemas dinámicos disipativos: uno continuo y otro discreto.

\section{Introducción}

La modelización de sistemas físicos es fundamental en una amplia variedad de disciplinas, tanto a nivel científico como empresarial, para predecir su futura evolución en el tiempo y poder tomar decisiones en base a esa información. Actualmente existen multitud de modelos que describen las leyes físicas de la naturaleza, desarrollados y validados mediante datos experimentales. Sin embargo, ciertos fenómenos son especialmente difíciles de modelar con fórmulas matemáticas exactas, como pueden ser los materiales viscoelasticos o fluidos no-Newtonianos.

Para resolver este problema, en las últimas décadas se han desarrollado técnicas basadas en datos (datadriven) que se aprovechan de los crecientes avances informáticos en gestión de datos para aprender los modelos directamente de observaciones y no depender de un modelo matemático cerrado. En concreto, las redes neuronales profundas (deep neural networks) han conseguido resultados excelentes en problemas tradicionalmente muy difíciles de resolver como el procesamiento del habla, detección automática de escritura o identificación facial.

Dentro del contexto de las simulaciones físicas, las redes neuronales han demostrado ser muy eficaces en la resolución de ecuaciones diferenciales en derivadas parciales de sistemas dinámicos [1]. Sin embargo, la mayoría de estas implementaciones parten de un conocimiento previo de las ecuaciones del sistema, que es precisamente lo que se desea modelar. Los primeros intentos de redes que son capaces de detectar verdaderamente la física interna del problema son las llamadas redes Hamiltonianas o simplécticas [2]. Estas redes se aprovechan del formalismo Hamiltoniano para aprender la dependencia entre la energía del sistema y la posición y el momento lineal de las partículas que lo componen. El mayor inconveniente de este método es que está restringido a sistemas conservativos, es decir, sin disipación de energía.

El objetivo de este trabajo es desarrollar un tipo de red neuronal capaz de predecir la evolución temporal de un sistema arbitrario (es decir, un integrador) sin saber a priori sus ecuaciones e incluyendo fenómenos de disipación.

\section{Metodología}

El trabajo se basa principalmente en el desarrollo de dos herramientas: una red encargada de identificar la estructura interna del sistema y de predecir su evolución en el tiempo y otra red cuyo objetivo es reducir el número de variables en caso de que el sistema tenga muchos grados de libertad.

\section{Red neuronal GENERIC}

Esta red realiza la tarea de integrador, que está forzado a identificar la estructura del sistema mediante el formalismo GENERIC (General Equation for Non-Equilibrium ReversibleIrreversible Coupling) [3]. La estructura GENERIC divide al sistema en dos contribuciones distintas: una conservativa, descrita por las ecuaciones de Hamilton mencionadas anteriormente, y otra disipativa, de tal forma que la evolución del vector de estados de un sistema (z) queda determinado por:

$$
\frac{d z}{d t}=L \frac{\partial E}{\partial z}+\boldsymbol{M} \frac{\partial S}{\partial z}
$$

Las matrices $\mathbf{L}$ y $\mathbf{M}$ codifican la relación entre el vector de estados $\mathbf{z}$ y la energía $\mathrm{E}$ y entropía $\mathrm{S}$ del sistema respectivamente. Para que estos operadores 
sean consistentes con las leyes de la termodinámica, se deben de asegurar varias condiciones adicionales: $\mathbf{L}$ es antisimétrica y $\mathbf{M}$ es simétrica y semi-definida positiva. Además, ambas deben cumplir las condiciones de degeneración:

$$
\boldsymbol{L} \frac{\partial S}{\partial \mathbf{z}}=\boldsymbol{M} \frac{\partial E}{\partial \mathbf{z}}=0
$$

Por tanto, la entrada de la red neuronal es el vector de estados en un instante de tiempo $\mathbf{z}(\mathrm{t})$ y la red calcula las matrices $\mathbf{L}$ y $\mathbf{M}$ y los gradientes $\partial E / \partial \mathbf{z}$ y $\partial S / \partial \mathbf{z}$ restringidos a todas las condiciones descritas, para luego hacer la predicción en el instante siguiente $\mathbf{z}(\mathrm{t}+1)$ mediante la fórmula (1).

\section{Reducción de dimensionalidad}

Para manejar sistemas con un gran número de grados de libertad, se ha desarrollado un tipo de autoencoder capaz de proyectar la dinámica del espacio original de alta dimensionalidad $\mathbb{R}^{D}$ en otro reducido en $\mathbb{R}^{d}$ con $d \ll D$. Además, aplicando una regularización sparse, el autoencoder es capaz de identificar automáticamente la dimensionalidad efectiva $(d)$ del modelo reducido sin necesidad de conocerla o imponerla de antemano.

\section{Resultados}

Para demostrar la efectividad del método, se han generado datos sintéticos para entrenar una red neuronal GENERIC en dos sistemas dinámicos distintos. El primero (Figura 1, izquierda) es un
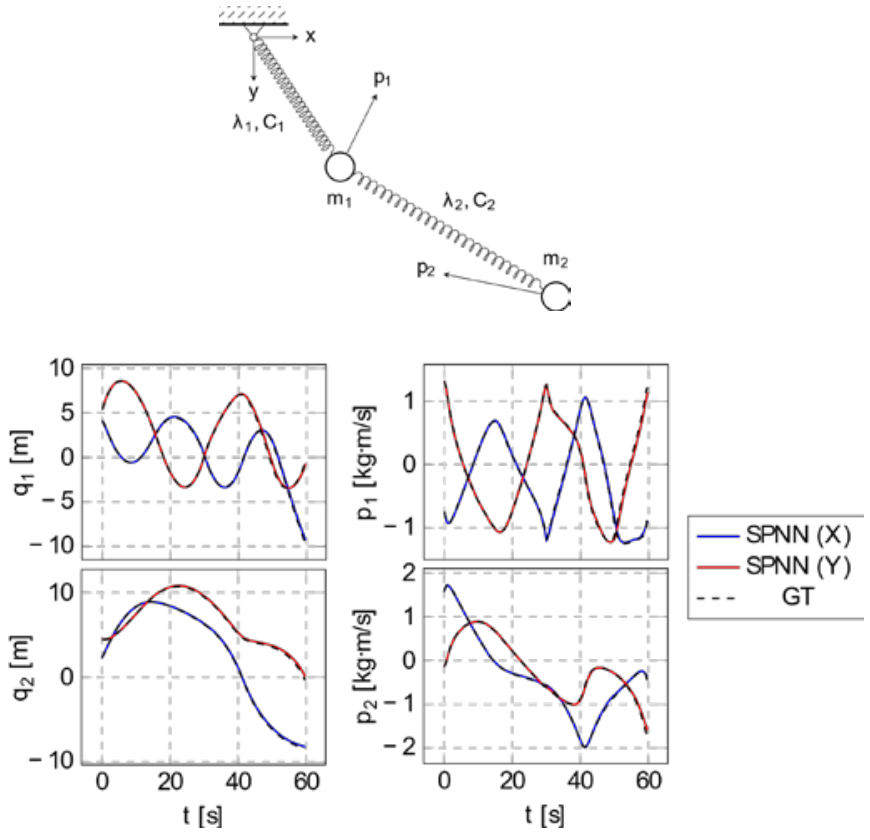

péndulo doble termoelástico, que incluye generación de entropía. El segundo (Figura 1, derecha) es un flujo de Couette de un fluido viscolástico de tipo Olroyd-B. En ambos casos el algoritmo es capaz de hacer predicciones estables sobre trayectorias no vistas durante el entrenamiento de la red [4].

\section{Conclusiones}

En este trabajo se demuestra como las redes neuronales GENERIC son capaces de aprender la estructura física de un sistema, y con ello realizar predicciones fiables y consistentes en el tiempo.

\section{REFERENCIAS}

[1]. RAISSI, M., PERDIKARIS, P. and KARNIADAKIS, G.E., 2019. Physics-informed neural networks: A deep learning framework for solving forward and inverse problems involving nonlinear partial differential equations. Journal of Computational Physics, 378, pp.686-707.

[2]. GREYDANUS, S., DZAMBA, M. and YOSINSKI, J., 2019. Hamiltonian neural networks. In Advances in Neural Information Processing Systems (pp. 1537915389).

[3]. GRMELA, M. and ÖTTINGER, H.C., 1997. Dynamics and thermodynamics of complex fluids. I. Development of a general formalism. Physical Review E, 56(6), p.6620.

[4]. HERNANDEZ, Q., BADIAS, A., GONZALEZ, D., CHINESTA, F. and CUETO, E., 2020. Structurepreserving neural networks. Journal of Computational Physics.
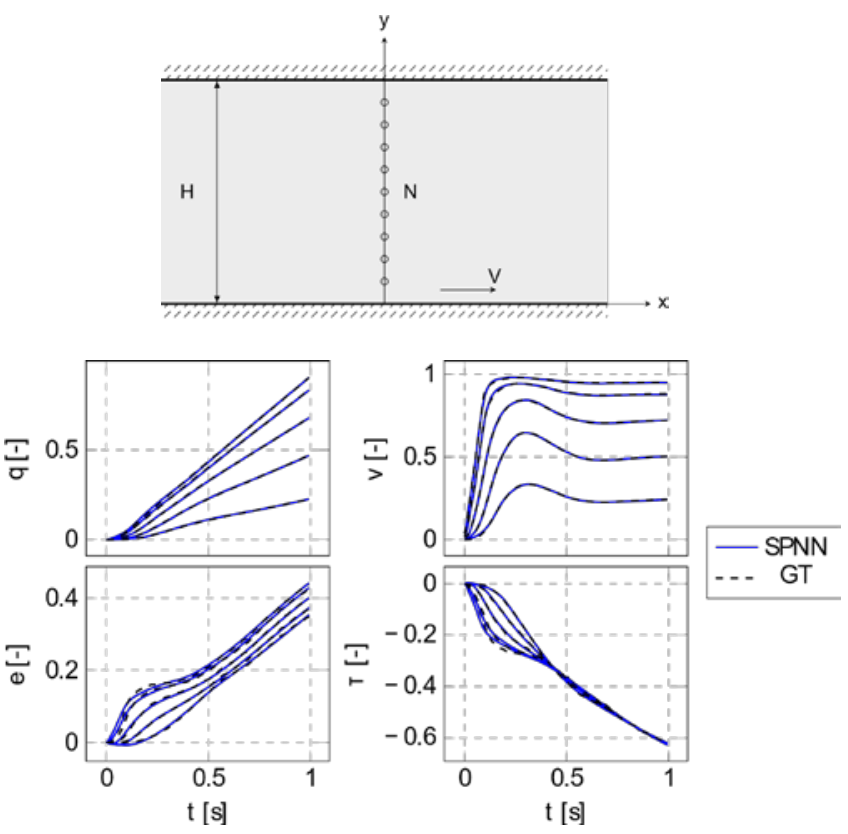

Figura 1. Trayectorias de validación de las variables de estados del péndulo doble (izquierda) y fluido viscolástico (derecha). GT: real (Ground Truth), SPNN: red neuronal (Structure-Preserving Neural Network).

Revista “Jornada de Jóvenes Investigadores del I3A”, vol. 8 (Actas de la IX Jornada de Jóvenes Investigadores del I3A -11 de diciembre de 2020). ISSN 2341-4790. 